\title{
Qualidade fisiológica de sementes de girassol avaliadas por condutividade elétrica ${ }^{1}$
}

\author{
Fabrícia Nascimento de Oliveira ${ }^{2}$, Salvador Barros Torres ${ }^{3}$, \\ Francisco Elvis Ramos Vieira ${ }^{2}$, Emanoela Pereira de Paiva ${ }^{2}$, Alek Sandro Dutra ${ }^{4}$
}

\begin{abstract}
Physiological quality of sunflower seeds according to electrical conductivity

The seed quality evaluation is essential in quality control programs. Thus, vigor tests have been developed to differentiate seed lots, due to the limitations of the germination test. This study aimed at evaluating the electrical conductivity test efficiency for determining the physiological quality of sunflower seeds. Ten lots of sunflower seeds (Catissol and Multissol cultivars) were used. Initially, the quality of each lot was determined, besides the seeds moisture content, by using tests of germination, first counting and seedling emergence, in a greenhouse. Then, the seeds were submitted to the electrical conductivity test, for four soaking periods $(6,12,18$ and 24 hours), by using disposable plastic cups containing 25 and 50 seeds, in $50 \mathrm{~mL}$ and $75 \mathrm{~mL}$ of deionized water, at $25^{\circ} \mathrm{C}$ and $30^{\circ} \mathrm{C}$. The experimental design was completely randomized, in a $5 \times 4$ factorial scheme (five lots and four soaking periods), separately for each cultivar, with four replications. It was possible to conclude that the electrical conductivity test combining $25^{\circ} \mathrm{C}, 75 \mathrm{~mL}$ of deionized water and 50 seeds was sufficiently sensitive to evaluate the physiological quality of sunflower seeds.
\end{abstract}

KEY-WORDS: Helianthus annuus L.; physiological potential; germination.

\section{INTRODUÇÃO}

O girassol (Helianthus annuus L.) está entre as cinco maiores culturas produtoras de óleo vegetal comestível do mundo, ficando apenas atrás da soja, algodão, colza e amendoim (Fagundes 2002). A demanda por sementes de girassol tem aumentado, devido à importância econômica do óleo extraído de seus aquênios, utilizado, principalmente, para consumo humano e animal, bem como matéria-prima para a produção de biocombustível (Ungaro 2006).

\section{RESUMO}

A avaliação da qualidade fisiológica de sementes é essencial em programas de controle de qualidade. Neste sentido, testes de vigor têm sido desenvolvidos para diferenciar lotes de sementes, devido às limitações do teste de germinação. Esta pesquisa objetivou avaliar a eficiência do teste de condutividade elétrica na determinação da qualidade fisiológica de sementes de girassol. Foram utilizados dez lotes de sementes de girassol das cultivares Catissol e Multissol. Inicialmente, determinou-se a qualidade dos lotes, mediante testes de germinação, primeira contagem e emergência de plântulas, em casa-de-vegetação, além do teor de água das sementes. Em seguida, as sementes foram submetidas ao teste de condutividade elétrica, em quatro períodos de embebição $(6,12,18$ e 24 horas), utilizando-se copos plásticos descartáveis contendo 25 e 50 sementes, em $50 \mathrm{~mL}$ e $75 \mathrm{~mL}$ de água deionizada, a $25^{\circ} \mathrm{C}$ e $30^{\circ} \mathrm{C}$. O delineamento utilizado foi o inteiramente casualizado, em esquema fatorial $5 \times 4$ (cinco lotes e quatro períodos de embebição), separadamente para cada cultivar, com quatro repetições. Concluiu-se que o teste de condutividade elétrica utilizando a combinação de $25^{\circ} \mathrm{C}, 75 \mathrm{~mL}$ de água deionizada e 50 sementes foi suficientemente sensível para avaliar a qualidade fisiológica das sementes de girassol.

PALAVRAS-CHAVE: Helianthus annuus L.; potencial fisiológico; germinação.

Segundo Scheeren et al. (2010), conhecer a qualidade das sementes antes da semeadura é o procedimento mais correto e seguro para se evitar aumentos no custo da lavoura. Pesquisas com o girassol, principalmente na área de controle de qualidade de sementes, são essenciais para o estabelecimento da cultura e se justificam pela potencialidade da espécie. Logo, torna-se imprescindível ajustar tecnologias para a produção de sementes, que permitam homogeneizar o processo de germinação, bem como reduzir o tempo de germinação entre lotes, facilitan-

1. Trabalho recebido em abr./2012 e aceito para publicação em ago./2012 ( ${ }^{\circ}$ registro: PAT 17793).

2. Universidade Federal Rural do Semiárido (Ufersa), Departamento de Ciências Vegetais, Mossoró, RN, Brasil. E-mails: fabricia@ufersa.edu.br, elvis.agro@hotmail.com, emanuelappaiva@hotmail.com.

3. Empresa de Pesquisa Agropecuária do Rio Grande do Norte (Emparn), Departamento de Ciências Vegetais, Mossoró, RN, Brasil.E-mail: sbtorres@ufersa.edu.br.

4. Universidade Federal do Ceará (UFC), Departamento de Fitotecnia, Fortaleza, CE, Brasil. E-mail: alekdutra@ufc.br. 
do tomadas de decisão, em relação à origem e ao destino dos lotes.

Atualmente, o procedimento utilizado para a determinação da qualidade de sementes de girassol é o teste de germinação (Brasil 2009), que não é eficiente para a diferenciação do vigor dos lotes, havendo necessidade da adoção de testes específicos (Marcos Filho 1999), dentre os quais o de condutividade elétrica, que é um meio rápido e prático para tal finalidade (Vieira \& Krzyzanowski 1999).

O teste de condutividade elétrica determina a quantidade de lixiviados na solução de embebição das sementes, sendo maior para sementes com menor vigor e menor para aquelas com maior vigor (AOSA 1983, Hampton \& Tekrony 1995). Este teste é recomendado para avaliar o vigor de sementes de ervilha (Nijênstein et al. 2007) e, também, sugerido para sementes de soja (Hampton \& Tekrony 1995).

O teste de condutividade elétrica já foi avaliado para as culturas do feijão (Krzyzanowski et al. 1999), café (Costa \& Carvalho 2006), soja (Krzyzanowski et al. 1999, Vieira et al. 2004, Colete et al. 2004), milho (Krzyzanowski et al. 1999, Alves et al. 2004), mamona (Souza et al. 2009), amendoim (Vanzolini \& Nakagawa 2005) e algodão (Torres 1998, Krzyzanowski et al. 1999). No entanto, para sementes de girassol, o teste de condutividade elétrica ainda carece de estudos que visem a aperfeiçoar a técnica para avaliação da qualidade fisiológica desta espécie, visto que as informações observadas na literatura, até o momento, mostram que o teste não foi eficiente, quando correlacionado com a emergência de plântulas em campo (Albuquerque et al. 2001).

Neste sentido, Queiroga \& Duran (1997), trabalhando com três cultivares de girassol, com diferentes graus de hidratação das sementes, avaliaram a sensibilidade do teste para a separação de lotes de alto e baixo vigor. Brandão Júnior et al. (1997), utilizando o teste de condutividade pelo sistema de massa, na avaliação de sementes íntegras e destegumentadas de girassol, cultivar IAC-Anhandy, verificaram que os tratamentos com sementes destegumentadas, embebidas por 18 e 24 horas, apresentaram maior eficiência para detectar as diferenças de qualidade existentes entre os lotes. Por outro lado, Albuquerque et al. (2001) e Abreu et al. (2011) verificaram que o teste de condutividade elétrica não foi eficiente para a separação de lotes de sementes de girassol, em diferentes níveis de vigor, e que não houve correlação significativa entre os resultados do teste de emergência de plântulas em campo e os resultados de condutividade elétrica.

Diante do exposto, este trabalho objetivou avaliar a metodologia do teste de condutividade elétrica para avaliação do vigor de sementes de girassol, cultivares Catissol e Multissol.

\section{MATERIAL E MÉTODOS}

O experimento foi desenvolvido no Laboratório de Análise de Sementes do Departamento de Ciências Vegetais e em casa-de-vegetação da Universidade Federal Rural do Semiárido, em Mossoró (RN), em 2011. Foram utilizados cinco lotes de duas cultivares de girassol (Catissol e Multissol), adquiridas junto à Coordenadoria de Assistência Técnica Integral (CATI), em Campinas (SP).

Durante oito meses (período experimental), as sementes permaneceram embaladas em sacos de papel Kraft multifoliado e armazenadas em ambiente com temperatura de $16-18^{\circ} \mathrm{C}$ e umidade relativa do ar de $45-50 \%$.

A qualidade fisiológica das sementes foi verificada mediante as seguintes avaliações:

a) Grau de umidade: expresso em percentagem média (base úmida) e avaliado a $105 \pm 3{ }^{\circ} \mathrm{C}$, durante 24 horas (Brasil 2009), utilizando-se duas subamostras de $4 \mathrm{~g}$ de sementes, para cada lote;

b) Teste de germinação: foram utilizadas 4 repetições de 50 sementes por lote, distribuídas em bandejas de areia, previamente umedecidas com quantidade de água equivalente a $60 \%$ da capacidade de campo, colocadas em bancadas e mantidas em temperatura ambiente $\left( \pm 27^{\circ} \mathrm{C}\right)$. As contagens foram efetuadas aos quatro e dez dias após a instalação do teste (Brasil 2009), sendo os resultados expressos em percentagem média de plântulas normais, para cada lote;

c) Primeira contagem de germinação: avaliada juntamente com o teste de germinação, computando-se a percentagem de plântulas normais, no quarto dia após a instalação do teste (Brasil 2009);

d) Emergência de plântulas em casa-de-vegetação: avaliada com quatro repetições de 50 sementes por lote, em bandejas de polietileno com 128 células, contendo substrato Plantimax ${ }^{\circledR}$, mantidas em ambiente protegido, com irrigação periódica e sob temperatura média de $30^{\circ} \mathrm{C}$. A avaliação da emergência das plântulas foi efetuada aos dez dias após a semeadura, mediante a contagem de plântulas normais emergi- 
das, sendo os resultados expressos em percentagem média, para cada lote (Nakagawa 1994);

e) Condutividade elétrica: determinou-se a liberação de eletrólitos, para cada lote, por meio da análise dos efeitos do período de embebição, volume de água destilada, temperatura e número de sementes, utilizando-se 25 e 50 sementes, colocadas em copos plásticos contendo $50 \mathrm{~mL}$ e $75 \mathrm{~mL}$ de água destilada e mantidas a $25^{\circ} \mathrm{C}$ e $30^{\circ} \mathrm{C}$, durante $6,12,18$ e 24 horas de embebição. As avaliações foram conduzidas com quatro repetições, utilizando-se sementes previamente pesadas, sem pericarpo (Albuquerque et al. 2001), embebidas em água destilada e mantidas em BOD, durante cada período considerado. A quantidade de lixiviados foi determinada com o auxílio de condutivímetro e os resultados expressos em $\mu \mathrm{S} \mathrm{cm}^{-1} \mathrm{~g}^{-1}$ de semente.

O experimento foi conduzido em delineamento estatístico inteiramente casualizado, em esquema fatorial $5 \times 4$ (cinco lotes e quatro períodos de embebição), com quatro repetições. A análise de variância foi efetuada separadamente para cada teste e cultivar. As médias obtidas das avaliações de cada lote, quando significativas pelo teste $\mathrm{F}$, foram comparadas pelo teste Tukey, a $5 \%$, enquanto as médias dos períodos de embebição foram avaliadas por meio de análise de regressão, ajustando-se modelos de equações lineares significativas pelo teste F. Para execução das análises estatísticas, foi utilizado o programa estatístico Sisvar (Ferreira 2008).

\section{RESULTADOS E DISCUSSÃO}

Não foram observadas grandes variações nos teores de umidade das sementes, entre os lotes e cultivares, ficando a cultivar Multissol com 6,2-6,7\% de água e a Catissol com 5,1-5,9\% (Tabela 1). A uniformidade deste parâmetro é essencial para a padronização das avaliações e obtenção de resultados consistentes (Krzyzanowski et al. 1991, Marcos Filho 1999).

Os lotes de sementes da cultivar Catissol avaliados não se distinguiram, quando avaliada a percentagem de germinação (Tabela 1), variando 92-97\%. Segundo Marcos Filho (1999), isto não significa que, em condições de campo, estes lotes terão as mesmas percentagens de germinação, uma vez que o teste de germinação é conduzido em condições favoráveis de laboratório. Entretanto, os testes de primeira contagem de germinação e emergência de plântulas
Tabela 1. Grau de umidade (GU), germinação total $(\mathrm{G})$, primeira contagem de germinação (PCG) e emergência de plântulas (EP), em casa-de-vegetação, para lotes de sementes de girassol das cultivares Catissol e Multissol (Mossoró, RN, 2011).

\begin{tabular}{|c|c|c|c|c|c|}
\hline \multirow{2}{*}{ Cultivar } & \multirow{2}{*}{ Lote } & GU & G & PCG & EP \\
\hline & & \multicolumn{4}{|c|}{$\%$} \\
\hline \multirow{6}{*}{ Catissol } & 1 & 5,0 & $92 a^{*}$ & $77 \mathrm{ab}$ & $91 \mathrm{ab}$ \\
\hline & 2 & 5,3 & $92 \mathrm{a}$ & $66 \mathrm{~b}$ & $85 \mathrm{~b}$ \\
\hline & 3 & 5,1 & $94 \mathrm{a}$ & $83 \mathrm{a}$ & 96 a \\
\hline & 4 & 5,0 & $97 \mathrm{a}$ & $77 \mathrm{ab}$ & $95 \mathrm{a}$ \\
\hline & 5 & 5,9 & $93 \mathrm{a}$ & $66 \mathrm{~b}$ & $88 \mathrm{ab}$ \\
\hline & CV (\%) & - & 7,2 & 4,7 & 4,2 \\
\hline \multirow{6}{*}{ Multissol } & 6 & 6,3 & $95 \mathrm{ab}$ & $59 \mathrm{ab}$ & $87 \mathrm{ab}$ \\
\hline & 7 & 6,2 & 99 a & $76 \mathrm{a}$ & $91 \mathrm{a}$ \\
\hline & 8 & 6,4 & $90 \mathrm{~b}$ & $40 \mathrm{bc}$ & $78 \mathrm{~b}$ \\
\hline & 9 & 6,7 & $95 \mathrm{ab}$ & $31 \mathrm{c}$ & $81 \mathrm{ab}$ \\
\hline & 10 & 6,2 & $95 \mathrm{ab}$ & $66 \mathrm{a}$ & $88 \mathrm{ab}$ \\
\hline & CV (\%) & - & 18,9 & 6,9 & 4,1 \\
\hline
\end{tabular}

* Letras minúsculas: comparação das médias em cada coluna (Tukey, $\mathrm{p} \leq 0,05$ ), separadamente para cada cultivar.

(Tabela 1), em casa-de-vegetação, apresentaram maior sensibilidade, indicando diferentes valores de vigor dos lotes 2 e 3 , respectivamente, em comparação aos demais lotes. O lote 7 da cultivar Multissol destacou-se quanto à percentagem de germinação, enquanto os lotes 6,9 e 10 não diferiram entre si, em relação à percentagem de germinação, e o lote 8 foi o que apresentou pior qualidade fisiológica.

Para as duas cultivares avaliadas, foi observada percentagem de germinação acima de $90 \%$ e emergência de plântulas acima de $81 \%$, indicando satisfatória qualidade fisiológica das sementes (Tabela 1).

Nas Tabelas 2 e 3, podem ser observados os resultados dos diferentes procedimentos utilizados no teste de condutividade elétrica, para as cultivares Catissol e Multissol, à temperatura de $25^{\circ} \mathrm{C}$.

Observa-se, na Tabela 2, que, nos diferentes procedimentos em que foram utilizadas 25 sementes embebidas em $50 \mathrm{~mL}$, não houve diferença estatística entre os lotes de baixo e alto vigor, exceto quando utilizou-se o período de embebição de 24 horas. Foi observado, ainda, que, em todas as combinações do teste de condutividade elétrica, os eletrólitos lixiviados pelas sementes aumentaram linearmente, durante a embebição, constatação feita, também, por Barbieri et al. (2012), com sementes de arroz.

Pesquisas têm evidenciado que, além do nível de deterioração e do efeito da temperatura, outros fatores diretamente relacionados às características das 
Tabela 2. Condutividade elétrica $\left(\mu \mathrm{S} \mathrm{cm}^{-1} \mathrm{~g}^{-1}\right)$, utilizando-se as combinações de 25 sementes $/ 50 \mathrm{~mL}$ e 50 sementes $/ 50 \mathrm{~mL}$, a $25^{\circ} \mathrm{C}$, de lotes de sementes de girassol das cultivares Catissol e Multissol, em cada período de embebição (Mossoró, RN, 2011).

\begin{tabular}{|c|c|c|c|c|c|c|c|}
\hline \multirow{2}{*}{ Cultivar } & \multirow{2}{*}{ Lote } & \multicolumn{4}{|c|}{ Período de embebição (h) } & \multirow{2}{*}{ Equação de regressão } & \multirow{2}{*}{$\mathrm{R}^{2}$} \\
\hline & & 6 & 12 & 18 & 24 & & \\
\hline \multirow{7}{*}{ Catissol } & & & 5 semente & $\mathrm{mL} / 25^{\circ} \mathrm{C}$ & +2 & \multirow{7}{*}{$\begin{array}{l}\text { Não significativa } \\
\text { Não significativa } \\
\text { Não significativa } \\
\text { Não significativa } \\
\mathrm{y}=34,95+1,9683 \mathrm{x} * *\end{array}$} & \multirow[b]{2}{*}{ - } \\
\hline & 1 & $47,9 \mathrm{~A}$ & $57,3 \mathrm{~A}$ & $66,1 \mathrm{~A}$ & $67,9 \mathrm{AB}$ & & \\
\hline & 2 & $36,7 \mathrm{~A}$ & $44,8 \mathrm{~A}$ & $53,7 \mathrm{~A}$ & $56,2 \mathrm{~A}$ & & - \\
\hline & 3 & $46,2 \mathrm{~A}$ & $57,6 \mathrm{~A}$ & $66,5 \mathrm{~A}$ & $68,3 \mathrm{AB}$ & & - \\
\hline & 4 & $35,7 \mathrm{~A}$ & $44,4 \mathrm{~A}$ & $51,5 \mathrm{~A}$ & $55,1 \mathrm{~A}$ & & - \\
\hline & 5 & $45,3 \mathrm{~A}$ & $60,2 \mathrm{~A}$ & $71,5 \mathrm{~A}$ & $80,9 \mathrm{~B}$ & & 0,98 \\
\hline & \multicolumn{5}{|c|}{$\mathrm{CV}(\%)=22,4$} & & \\
\hline \multirow{6}{*}{ Multissol } & 6 & $42,8 \mathrm{BC}$ & $54,7 \mathrm{BC}$ & $63,5 \mathrm{~B}$ & $68,9 \mathrm{~B}$ & $\mathrm{y}=35,7+1,4517 \mathrm{x}^{* *}$ & 0,97 \\
\hline & 7 & $58,0 \mathrm{C}$ & $71,2 \mathrm{C}$ & $78,2 \mathrm{~B}$ & $81,5 \mathrm{~B}$ & $\mathrm{y}=52,85+1,2917 \mathrm{x} * *$ & 0,92 \\
\hline & 8 & $22,7 \mathrm{~A}$ & $26,9 \mathrm{~A}$ & $28,4 \mathrm{~A}$ & $29,1 \mathrm{~A}$ & Não significativa & - \\
\hline & 9 & $32,2 \mathrm{AB}$ & $39,5 \mathrm{AB}$ & $43,9 \mathrm{~A}$ & $44,8 \mathrm{~A}$ & Não significativa & - \\
\hline & 10 & $48,0 \mathrm{BC}$ & $57,9 \mathrm{BC}$ & $65,9 \mathrm{~B}$ & $68,3 \mathrm{~B}$ & $y=42,8+1,1483 x^{* *}$ & 0,94 \\
\hline & \multicolumn{5}{|c|}{$\mathrm{CV}(\%)=19,0$} & \multirow{8}{*}{$\begin{array}{l}\mathrm{y}=41,65+1,3417 \mathrm{x} * * \\
\mathrm{y}=36,45+1,2917 \mathrm{x} * * \\
\mathrm{y}=54+1,7683 \mathrm{x} * * \\
\text { Não significativa } \\
\text { Não significativa }\end{array}$} & \\
\hline \multirow{7}{*}{ Catissol } & & & 0 semente & $\mathrm{mL} / 25^{\circ} \mathrm{C}$ & 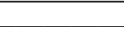 & & \multirow{7}{*}{$\begin{array}{c}0,93 \\
0,97 \\
0,97 \\
- \\
-\end{array}$} \\
\hline & 1 & $47,3 \mathrm{BC}$ & $60,2 \mathrm{~B}$ & $68,1 \mathrm{C}$ & $71,5 \mathrm{C}$ & & \\
\hline & 2 & $42,5 \mathrm{AB}$ & $53,9 \mathrm{AB}$ & $60,9 \mathrm{BC}$ & $66,0 \mathrm{BC}$ & & \\
\hline & 3 & $62,3 \mathrm{C}$ & $77,7 \mathrm{C}$ & $87,8 \mathrm{D}$ & $94,3 \mathrm{D}$ & & \\
\hline & 4 & $30,4 \mathrm{~A}$ & $38,4 \mathrm{~A}$ & $42,9 \mathrm{~A}$ & $45,3 \mathrm{~A}$ & & \\
\hline & 5 & $37,1 \mathrm{AB}$ & $45,3 \mathrm{AB}$ & $50,5 \mathrm{AB}$ & $51,9 \mathrm{AB}$ & & \\
\hline & \multicolumn{5}{|c|}{$\mathrm{CV}(\%)=14,9$} & & \\
\hline \multirow{6}{*}{ Multissol } & 6 & $31,2 \mathrm{~B}$ & $36,5 \mathrm{~B}$ & $42,6 \mathrm{~B}$ & $48,7 \mathrm{~B}$ & $\mathrm{y}=25,1+0,9767 x^{* *}$ & 0,99 \\
\hline & 7 & $49,7 \mathrm{C}$ & $60,4 \mathrm{D}$ & $69,2 \mathrm{D}$ & $75,6 \mathrm{C}$ & $\mathrm{y}=42,1+1,4417 \mathrm{x} * *$ & 0,98 \\
\hline & 8 & $17,9 \mathrm{~A}$ & $21,6 \mathrm{~A}$ & $24,4 \mathrm{~A}$ & $25,9 \mathrm{~A}$ & Não significativa & - \\
\hline & 9 & $28,6 \mathrm{~B}$ & $36,4 \mathrm{~B}$ & $42,6 \mathrm{~B}$ & $47,9 \mathrm{~B}$ & \multirow{3}{*}{$\begin{array}{l}\mathrm{y}=22,85+1,0683 \mathrm{x}^{* *} \\
\mathrm{y}=31,4+1,1367 \mathrm{x}^{* *}\end{array}$} & 0,99 \\
\hline & 10 & $36,9 \mathrm{~B}$ & $46,2 \mathrm{C}$ & $53,5 \mathrm{C}$ & $57,2 \mathrm{~B}$ & & \multirow{2}{*}{0,97} \\
\hline & & & $\mathrm{CV}(\%)=$ & & & & \\
\hline
\end{tabular}

Médias seguidas da mesma letra maiúscula, na coluna, não diferem entre si, pelo teste Tukey (p $\leq 0,05)$. ${ }^{*}$ Significativo a $5 \%$ (Teste F).

sementes, ou a própria metodologia, podem interferir nos resultados do teste de condutividade, tais como a qualidade da água, duração do período de hidratação e o tamanho, grau de umidade e número de sementes testadas (Dias \& Marcos Filho 1996, Oliveira \& Novembre 2005). Para a cultivar Catissol, as combinações de número de sementes e volume de água, nos diferentes períodos de embebição, mostraram-se eficientes na diferenciação da qualidade fisiológica entre os lotes estudados, indicando o lote 4 como de qualidade superior aos demais. Esta tendência é a mesma obtida no teste de emergência de plântulas em casa-de-vegetação (Tabela 1).

De maneira geral, observou-se que, a $30^{\circ} \mathrm{C}$, o aumento nos períodos de embebição provocou diminuição linear na condutividade elétrica (Tabelas 4 e 5).

As diversas combinações de número de sementes e volumes de água, para as temperaturas de $25^{\circ} \mathrm{C}$ e $30^{\circ} \mathrm{C}$, apresentaram similaridade na determinação do vigor de lotes de sementes da cultivar Multissol, indicando o lote 8 como o de maior vigor e o lote 7 como o de menor vigor. Estes resultados contrariam os obtidos nos testes de germinação e emergência de plântulas em casa-de-vegetação (Tabela 1), que, em linhas gerais, apontaram o inverso, sendo o lote 7 superior e o lote 8 inferior aos demais. Informações semelhantes foram relatadas por Albuquerque et al. (2001), ao observarem que houve efeito dos genótipos de girassol no teste de condutividade elétrica, com inversão na sua classificação, em relação aos testes de avaliação da qualidade fisiológica inicial. De maneira semelhante, Vanzolini \& Nakagawa (1999) verificaram, em sementes de amendoim, que o teste de condutividade elétrica apontou inversão da qualidade dos lotes, em relação aos dados de emergência de plântulas.

Com relação ao período de embebição das sementes, verificou-se, nas diversas combinações, que houve aumento progressivo nas leituras, à medida em 
Tabela 3. Condutividade elétrica $\left(\mu \mathrm{S} \mathrm{cm} \mathrm{g}^{-1}\right)$, utilizando-se as combinações de 25 sementes $/ 75 \mathrm{~mL}$ e 50 sementes/ $75 \mathrm{~mL}$, a $25^{\circ} \mathrm{C}$, de lotes de sementes de girassol das cultivares Catissol e Multissol, em cada período de embebição (Mossoró, RN, 2011).

\begin{tabular}{|c|c|c|c|c|c|c|c|}
\hline \multirow{2}{*}{ Cultivar } & \multirow{2}{*}{ Lote } & \multicolumn{4}{|c|}{ Período de embebição (h) } & \multirow{2}{*}{ Equação de regressão } & \multirow{2}{*}{$\mathrm{R}^{2}$} \\
\hline & & 6 & 12 & 18 & 24 & & \\
\hline \multirow{7}{*}{ Catissol } & & & sementes/ & $\mathrm{nL} / 25^{\circ} \mathrm{C}$ & & \multirow[b]{2}{*}{$y=41,4+1,945 x^{* *}$} & \multirow[b]{2}{*}{0,98} \\
\hline & 1 & $51,5 \mathrm{C}$ & $66,1 \mathrm{~B}$ & $78,4 \mathrm{~B}$ & $86,3 \mathrm{~B}$ & & \\
\hline & 2 & $26,5 \mathrm{AB}$ & $33,5 \mathrm{~A}$ & $38,5 \mathrm{~A}$ & $41,1 \mathrm{~A}$ & Não significativa & - \\
\hline & 3 & $42,9 \mathrm{BC}$ & $62,4 \mathrm{~B}$ & $74,4 \mathrm{~B}$ & $80,5 \mathrm{~B}$ & $\mathrm{y}=33,85+2,08 x^{* *}$ & 0,94 \\
\hline & 4 & $22,9 \mathrm{~A}$ & $28,6 \mathrm{~A}$ & $32,9 \mathrm{~A}$ & $35,3 \mathrm{~A}$ & Não significativa & - \\
\hline & 5 & $28,9 \mathrm{AB}$ & $32,5 \mathrm{~A}$ & $35,7 \mathrm{~A}$ & $38,5 \mathrm{~A}$ & Não significativa & - \\
\hline & \multicolumn{5}{|c|}{$\mathrm{CV}(\%)=21,4$} & & \\
\hline \multirow{6}{*}{ Multissol } & 6 & $29,5 \mathrm{AB}$ & $36,7 \mathrm{~A}$ & $40,4 \mathrm{~A}$ & $43,3 \mathrm{AB}$ & Não significativa & - \\
\hline & 7 & $48,4 \mathrm{~B}$ & $65,6 \mathrm{~B}$ & $63,7 \mathrm{~B}$ & $64,5 \mathrm{~B}$ & Não significativa & - \\
\hline & 8 & $19,6 \mathrm{~A}$ & $22,7 \mathrm{~A}$ & $23,9 \mathrm{~A}$ & $24,5 \mathrm{~A}$ & Não significativa & - \\
\hline & 9 & $29,9 \mathrm{AB}$ & $37,3 \mathrm{~A}$ & $44,2 \mathrm{AB}$ & $44,9 \mathrm{AB}$ & Não significativa & - \\
\hline & 10 & $31,7 \mathrm{AB}$ & $37,4 \mathrm{~A}$ & $43,4 \mathrm{AB}$ & $43,8 \mathrm{AB}$ & \multirow[t]{2}{*}{ Não significativa } & \multirow[t]{2}{*}{-} \\
\hline & \multicolumn{5}{|c|}{$\mathrm{CV}(\%)=28,4$} & & \\
\hline \multirow{7}{*}{ Catissol } & & & sementes/ & $\mathrm{nL} / 25^{\circ} \mathrm{C}$ & - & \multirow{7}{*}{$\begin{array}{l}\mathrm{y}=31,55+1,0883 \mathrm{x}^{* *} \\
\mathrm{y}=21+0,7033 \mathrm{x}^{* *} \\
\mathrm{y}=39,7+1,2667 \mathrm{x}^{* *} \\
\mathrm{y}=18,85+0,5533 \mathrm{x}^{* *} \\
\mathrm{y}=27+0,9617 \mathrm{x}^{* *}\end{array}$} & \multirow{7}{*}{$\begin{array}{l}0,98 \\
0,97 \\
0,97 \\
0,95 \\
0,97\end{array}$} \\
\hline & 1 & $36,9 \mathrm{CD}$ & $46,1 \mathrm{C}$ & $51,7 \mathrm{~B}$ & $56,8 \mathrm{~B}$ & & \\
\hline & 2 & $24,3 \mathrm{AB}$ & $30,6 \mathrm{AB}$ & $34,1 \mathrm{~A}$ & $37,2 \mathrm{~A}$ & & \\
\hline & 3 & $45,7 \mathrm{D}$ & $56,9 \mathrm{D}$ & $63,3 \mathrm{C}$ & $68,9 \mathrm{C}$ & & \\
\hline & 4 & $21,3 \mathrm{~A}$ & $26,5 \mathrm{~A}$ & $29,4 \mathrm{~A}$ & $31,4 \mathrm{~A}$ & & \\
\hline & 5 & $31,6 \mathrm{BC}$ & $39,7 \mathrm{BC}$ & $45,5 \mathrm{~B}$ & $48,9 \mathrm{~B}$ & & \\
\hline & & & $V(\%)=1$ & & & & \\
\hline \multirow{6}{*}{ Multissol } & 6 & $24,5 \mathrm{~B}$ & $30,6 \mathrm{~B}$ & $33,7 \mathrm{~B}$ & $36,7 \mathrm{~B}$ & $\mathrm{y}=21,45+0,6617 \mathrm{x} * *$ & 0,97 \\
\hline & 7 & $50,0 \mathrm{C}$ & $59,9 \mathrm{C}$ & $69,7 \mathrm{C}$ & 74,7 C & $y=42,6+1,3983 x * *$ & 0,98 \\
\hline & 8 & $13,1 \mathrm{~A}$ & $15,4 \mathrm{~A}$ & $16,1 \mathrm{~A}$ & $17,1 \mathrm{~A}$ & Não significativa & - \\
\hline & 9 & $22,7 \mathrm{AB}$ & $28,3 \mathrm{~B}$ & $31,5 \mathrm{~B}$ & $35,2 \mathrm{~B}$ & \multirow{3}{*}{$\begin{array}{l}y=19,25+0,6783 x^{* *} \\
y=27,15+0,755 x^{* *}\end{array}$} & 0,98 \\
\hline & 10 & $30,8 \mathrm{~B}$ & $37,9 \mathrm{~B}$ & $40,0 \mathrm{~B}$ & $45,2 \mathrm{~B}$ & & \multirow[t]{2}{*}{0,96} \\
\hline & & & $V(\%)=1$ & & & & \\
\hline
\end{tabular}

Médias seguidas da mesma letra maiúscula, na coluna, não diferem entre si, pelo teste Tukey $(\mathrm{p} \leq 0,05)$. ** Significativo a $5 \%$ (Teste F).

que se aumentou o período de embebição, de acordo com Loeffler et al. (1988), Marcos Filho et al. (1990), Dias et al. (1996) e Albuquerque et al. (2001).

Para o período de embebição de 6 horas, em todas as combinações avaliadas, foi possível a estratificação do vigor dos lotes das duas cultivares estudadas, exceto para a combinação de 25 sementes, $50 \mathrm{~mL}$ e $25^{\circ} \mathrm{C}$ da cultivar Catissol, que não permitiu esta separação. No entanto, esta estratificação só foi considerada consistente após o período de 16 horas de embebição, demonstrando, com confiança, a possibilidade de redução no período de embebição das sementes, em relação ao período de 24 horas, estabelecido como padrão.

Resultados semelhantes foram encontrados por Marcos Filho et al. (1990) e Dias \& Marcos Filho (1996), em soja; Vanzolini \& Nakagawa (1999), em amendoim; e Brandão Júnior et al. (1997), em sementes de girassol, cultivar IAC-Anhandy, que verificaram que as embebições por 18 e 24 horas foram mais eficientes para detectar as diferenças de vigor entre os lotes. Silva \& Martins (2009), em sementes de mamona, avaliaram a possibilidade da redução do tempo de embebição para 4 horas, com 25 sementes, e 6 horas, com 50 sementes. Araújo et al. (2011) observaram, em sementes de pinhão-manso, que a classificação dos lotes foi mais consistente após o período de 6 horas, o que representa significativa redução no período de condicionamento das sementes. Conforme Marcos Filho et al. (1990), testes que possibilitem a redução do período de avaliação assumem elevada importância para a indústria de sementes, pelo fato de reduzirem o período de tomada de decisão.

Em relação ao número de sementes, verificou-se que, quando se mantiveram constantes os outros parâmetros avaliados, à medida em que se aumentou o número de sementes, os resultados sofreram pequenos decréscimos nos valores de condutividade, para as duas cultivares. Neste sentido, constatou-se que 
Tabela 4. Condutividade elétrica $\left(\mu \mathrm{S} \mathrm{cm}^{-1} \mathrm{~g}^{-1}\right)$, utilizando-se as combinações de 25 sementes $/ 50 \mathrm{~mL}$ e 50 sementes $/ 50 \mathrm{~mL}$, a $30^{\circ} \mathrm{C}$, de lotes de sementes de girassol das cultivares Catissol e Multissol, em cada período de embebição (Mossoró, RN, 2011).

\begin{tabular}{|c|c|c|c|c|c|c|c|}
\hline \multirow{2}{*}{ Cultivar } & \multirow{2}{*}{ Lote } & \multicolumn{4}{|c|}{ Período de embebição (h) } & \multirow{2}{*}{ Equação de regressão } & \multirow{2}{*}{$\mathrm{R}^{2}$} \\
\hline & & 6 & 12 & 18 & 24 & & \\
\hline \multirow{7}{*}{ Catissol } & & & 25 sementes & $\mathrm{mL} / 30^{\circ} \mathrm{C}$ & & \multirow[b]{2}{*}{$\mathrm{y}=53,35+2,0533 \mathrm{x}^{* *}$} & \multirow[b]{2}{*}{0,96} \\
\hline & 1 & $63,1 \mathrm{~B}$ & $82,4 \mathrm{~B}$ & $89,2 \mathrm{C}$ & $101,9 \mathrm{C}$ & & \\
\hline & 2 & $46,5 \mathrm{AB}$ & $60,7 \mathrm{~A}$ & $66,2 \mathrm{AB}$ & $74,9 \mathrm{AB}$ & $\mathrm{y}=39,4+1,5117 \mathrm{x}^{* *}$ & 0,97 \\
\hline & 3 & $48,7 \mathrm{AB}$ & $61,6 \mathrm{~A}$ & $66,8 \mathrm{AB}$ & $74,2 \mathrm{AB}$ & $y=42,4+1,3617 x^{* *}$ & 0,96 \\
\hline & 4 & $39,2 \mathrm{~A}$ & $50,1 \mathrm{~A}$ & $53,9 \mathrm{~A}$ & $59,8 \mathrm{~A}$ & $y=34,35+1,0933 x^{* *}$ & 0,95 \\
\hline & 5 & $51,4 \mathrm{AB}$ & $68,4 \mathrm{AB}$ & $75,8 \mathrm{BC}$ & $85,4 \mathrm{BC}$ & $y=42,9+1,8233 x^{* *}$ & 0,97 \\
\hline & \multicolumn{5}{|c|}{$\mathrm{CV}(\%)=15,8$} & & \\
\hline \multirow{6}{*}{ Multissol } & 6 & $38,2 \mathrm{AB}$ & $47,6 \mathrm{AB}$ & $56,9 \mathrm{~B}$ & $61,4 \mathrm{~B}$ & Não significativa & - \\
\hline & 7 & $60,9 \mathrm{~B}$ & $74,3 \mathrm{C}$ & $87,5 \mathrm{C}$ & $100,3 \mathrm{C}$ & $y=47,9+2,19 x * *$ & 0,99 \\
\hline & 8 & $24,5 \mathrm{~A}$ & $28,2 \mathrm{~A}$ & $31,2 \mathrm{~A}$ & $33,5 \mathrm{~A}$ & Não significativa & - \\
\hline & 9 & $41,6 \mathrm{AB}$ & $53,7 \mathrm{ABC}$ & $62,4 \mathrm{BC}$ & $62,3 \mathrm{~B}$ & Não significativa & - \\
\hline & 10 & $48,2 \mathrm{AB}$ & $57,8 \mathrm{BC}$ & $63,5 \mathrm{BC}$ & $70,4 \mathrm{~B}$ & Não significativa & - \\
\hline & \multicolumn{5}{|c|}{$\mathrm{CV}(\%)=23,4$} & \multirow{8}{*}{$\begin{array}{l}\mathrm{y}=48,35+2,235 \mathrm{x}^{* *} \\
\mathrm{y}=30,9+1,35 \mathrm{x}^{* *} \\
\mathrm{y}=53,85+2,4567 \mathrm{x} * * \\
\mathrm{y}=26,6+0,8983 \mathrm{x} * * \\
\mathrm{y}=34,15+0,9917 \mathrm{x}^{* *}\end{array}$} & \\
\hline \multirow{7}{*}{ Catissol } & & & 50 sementes & $\mathrm{mL} / 30^{\circ} \mathrm{C}$ & & & \\
\hline & 1 & $60,6 \mathrm{~B}$ & $78,1 \mathrm{~B}$ & 86,2 B & $102,6 \mathrm{C}$ & & 0,98 \\
\hline & 2 & $38,1 \mathrm{~A}$ & $49,1 \mathrm{~A}$ & $53,9 \mathrm{Ab}$ & $63,5 \mathrm{~B}$ & & 0,98 \\
\hline & 3 & $67,1 \mathrm{~B}$ & $86,8 \mathrm{~B}$ & $95,6 \mathrm{~B}$ & $113,3 \mathrm{C}$ & & 0,98 \\
\hline & 4 & $30,8 \mathrm{~A}$ & $39,3 \mathrm{~A}$ & $42,5 \mathrm{~A}$ & $47,7 \mathrm{~A}$ & & 0,96 \\
\hline & 5 & $38,3 \mathrm{~A}$ & $48,9 \mathrm{~A}$ & $51,7 \mathrm{~A}$ & $57,2 \mathrm{AB}$ & & 0,94 \\
\hline & & & $\mathrm{CV}(\%)=$ & & & & \\
\hline \multirow{6}{*}{ Multissol } & 6 & $36,5 \mathrm{~B}$ & $45,9 \mathrm{BC}$ & $52,0 \mathrm{~B}$ & $59,7 \mathrm{~B}$ & \multirow{6}{*}{$\begin{array}{l}\mathrm{y}=29,6+1,2617 \mathrm{x} * * \\
\mathrm{y}=48,3+2,485 \mathrm{x} * * \\
\text { Não significativa } \\
\mathrm{y}=22,4+1,805 \mathrm{x} * * \\
\mathrm{y}=34,55+1,7017 \mathrm{x} * *\end{array}$} & 0,99 \\
\hline & 7 & $64,2 \mathrm{C}$ & $76,9 \mathrm{D}$ & $92,5 \mathrm{C}$ & $108,7 \mathrm{D}$ & & 0,99 \\
\hline & 8 & $22,0 \mathrm{~A}$ & $26,4 \mathrm{~A}$ & $28,7 \mathrm{~A}$ & $30,8 \mathrm{~A}$ & & - \\
\hline & 9 & $32,7 \mathrm{AB}$ & $44,7 \mathrm{~B}$ & $55,2 \mathrm{~B}$ & $65,3 \mathrm{BC}$ & & 0,99 \\
\hline & 10 & $41,9 \mathrm{~B}$ & $59,1 \mathrm{Cb}$ & $65,5 \mathrm{~B}$ & $73,8 \mathrm{C}$ & & 0,95 \\
\hline & & & $\mathrm{CV}(\%)=$ & & & & \\
\hline
\end{tabular}

Médias seguidas da mesma letra maiúscula, na coluna, não diferem entre si, pelo teste Tukey (p $\leq 0,05)$. ** Significativo a $5 \%$ (Teste F).

as combinações de 25 sementes, para as duas cultivares, apresentaram coeficiente de variação médio de $20,9 \%$, enquanto, para as de 50 sementes, foi de $13,3 \%$, recomendando-se o uso de $4 \times 50$ sementes, pois o coeficiente de variação foi menor do que quando foram utilizadas 25 sementes, corroborando os resultados obtidos por Loeffler et al. (1988).

O aumento no volume de água, quando se mantiveram constantes os outros fatores (temperatura, número de sementes e período de embebição), estabeleceu, em geral, relação direta com a diminuição no valor da lixiviação. Com volume de $50 \mathrm{~mL}$, foram observados os maiores valores de condutividade elétrica e, à medida em que houve aumento no volume de água utilizado, os valores da condutividade foram diminuindo gradativamente. Estes resultados foram semelhantes aos observados por Loeffler et al. (1988). Para os volumes estudados $(50 \mathrm{~mL}$ e $75 \mathrm{~mL})$, houve relação indireta com a estratificação, verificada nos testes iniciais de ava- liação do vigor das sementes da cultivar Multissol, apontando o lote 7 como o menos vigoroso e o lote 8 como o mais vigoroso.

A lixiviação de exsudatos foi proporcional ao aumento da temperatura empregada (de $25^{\circ} \mathrm{C}$ para $30^{\circ} \mathrm{C}$ ), mas manteve as tendências ordenadas de acréscimo dos lixiviados durante os períodos de embebição. Conforme Hampton \& Tekrony (1995), o efeito da temperatura é outro fator que se manifesta, basicamente, sobre a quantidade e velocidade de liberação de exsudatos, durante a embebição, sem alterar, necessariamente, a classificação dos lotes, quanto ao vigor. Nas sementes das grandes culturas, como a soja, verificou-se aumento da lixiviação até 24-30 horas após o início da embebição, a $25^{\circ} \mathrm{C}$ (Loeffler et al. 1988). Halder \& Gupta (1980) constataram que aquênios de girassol armazenados por mais de 90 dias, com umidade relativa elevada e temperaturas de $25^{\circ} \mathrm{C}$, provocaram aumento na lixiviação de eletrólitos. 
Tabela 5. Condutividade elétrica $\left(\mu \mathrm{S} \mathrm{cm}^{-1} \mathrm{~g}^{-1}\right)$, utilizando-se as combinações de 25 sementes $/ 75 \mathrm{~mL}$ e 50 sementes/ $75 \mathrm{~mL}$, a $30^{\circ} \mathrm{C}$, de lotes de sementes de girassol das cultivares Catissol e Multissol, em cada período de embebição (Mossoró, RN, 2011).

\begin{tabular}{|c|c|c|c|c|c|c|c|}
\hline \multirow{2}{*}{ Cultivar } & \multirow{2}{*}{ Lote } & \multicolumn{4}{|c|}{ Períodos de embebição $(\mathrm{h})$} & \multirow{2}{*}{ Equação de regressão } & \multirow{2}{*}{$\mathrm{R}^{2}$} \\
\hline & & 6 & 12 & 18 & 24 & & \\
\hline \multirow{7}{*}{ Catissol } & & & sementes & $\mathrm{nL} / 30^{\circ} \mathrm{C}$ & & \multirow{7}{*}{$\begin{array}{l}\mathrm{y}=42,4+1,4767 \mathrm{x} * * \\
\text { Não significativa } \\
\mathrm{y}=39,15+1,2983 \mathrm{x}^{* *} \\
\mathrm{y}=29,7+0,8317 \mathrm{x}^{* *} \\
\text { Não significativa }\end{array}$} & \multirow[b]{2}{*}{0,96} \\
\hline & 1 & $49,3 \mathrm{C}$ & $63,7 \mathrm{~B}$ & $67,7 \mathrm{~B}$ & $77,5 \mathrm{~B}$ & & \\
\hline & 2 & $27,8 \mathrm{~A}$ & $34,9 \mathrm{~A}$ & $36,3 \mathrm{~A}$ & $39,3 \mathrm{~A}$ & & - \\
\hline & 3 & $44,5 \mathrm{BC}$ & $58,1 \mathrm{~B}$ & $63,1 \mathrm{~B}$ & $68,8 \mathrm{~B}$ & & 0,94 \\
\hline & 4 & $32,9 \mathrm{AB}$ & $42,4 \mathrm{~A}$ & $44,6 \mathrm{~A}$ & $48,8 \mathrm{~A}$ & & 0,92 \\
\hline & 5 & $35,8 \mathrm{ABC}$ & $43,6 \mathrm{~A}$ & $45,0 \mathrm{~A}$ & $48,5 \mathrm{~A}$ & & - \\
\hline & \multicolumn{5}{|c|}{$\mathrm{CV}(\%)=14,7$} & & \\
\hline \multirow{6}{*}{ Multissol } & 6 & $23,9 \mathrm{~A}$ & $29,2 \mathrm{AB}$ & $32,5 \mathrm{AB}$ & $35,4 \mathrm{AB}$ & Não significativa & - \\
\hline & 7 & $45,8 \mathrm{C}$ & $58,6 \mathrm{C}$ & $67,8 \mathrm{C}$ & $75,9 \mathrm{C}$ & $\mathrm{y}=37,15+1,6583 \mathrm{x} * *$ & 0,98 \\
\hline & 8 & $14,2 \mathrm{~A}$ & $16,8 \mathrm{~A}$ & $18,5 \mathrm{~A}$ & $19,6 \mathrm{~A}$ & Não significativa & - \\
\hline & 9 & $26,6 \mathrm{AB}$ & 34,9 B & $39,2 \mathrm{~B}$ & $43,7 \mathrm{~B}$ & $y=22,2+0,9267 x * *$ & 0,97 \\
\hline & 10 & $42,0 \mathrm{BC}$ & $53,7 \mathrm{C}$ & $59,1 \mathrm{C}$ & $63,3 \mathrm{C}$ & $\mathrm{y}=37,2+1,155 \mathrm{x} * *$ & 0,94 \\
\hline & \multicolumn{6}{|c|}{$\mathrm{CV}(\%)=21,7$} & \\
\hline \multirow{7}{*}{ Catissol } & & 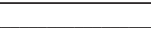 & sementes & $\mathrm{nL} / 30^{\circ} \mathrm{C}$ & 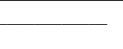 & \multirow{7}{*}{$\begin{array}{l}\mathrm{y}=30,45+1,1733 \mathrm{x}^{* *} \\
\mathrm{y}=22,55+0,7617 \mathrm{x}^{* *} \\
\mathrm{y}=35,1+1,4567 \mathrm{x}^{* *} \\
\mathrm{y}=22,75+0,71 \mathrm{x}^{* *} \\
\mathrm{y}=27,55+0,8383 \mathrm{x}^{* *}\end{array}$} & \multirow{7}{*}{$\begin{array}{l}0,97 \\
0,95 \\
0,98 \\
0,96 \\
0,94\end{array}$} \\
\hline & 1 & $36,5 \mathrm{AB}$ & $46,6 \mathrm{BC}$ & $50,4 \mathrm{BC}$ & $58,7 \mathrm{BC}$ & & \\
\hline & 2 & $26,1 \mathrm{~A}$ & $33,7 \mathrm{AB}$ & $35,3 \mathrm{~A}$ & $40,8 \mathrm{~A}$ & & \\
\hline & 3 & $42,7 \mathrm{~B}$ & $54,9 \mathrm{C}$ & $60,1 \mathrm{C}$ & $70,1 \mathrm{C}$ & & \\
\hline & 4 & $26,1 \mathrm{~A}$ & $32,9 \mathrm{~A}$ & $35,0 \mathrm{~A}$ & $39,6 \mathrm{~A}$ & & \\
\hline & 5 & $31,2 \mathrm{AB}$ & $40,0 \mathrm{AB}$ & $42,0 \mathrm{AB}$ & $47,3 \mathrm{AB}$ & & \\
\hline & \multicolumn{5}{|c|}{$\mathrm{CV}(\%)=15,8$} & & \\
\hline \multirow{6}{*}{ Multissol } & 6 & $40,9 \mathrm{~B}$ & $38,0 \mathrm{~B}$ & $43,3 \mathrm{~B}$ & $44,3 \mathrm{~B}$ & Não significativa & - \\
\hline & 7 & $47,0 \mathrm{~B}$ & $41,7 \mathrm{BC}$ & $47,4 \mathrm{~B}$ & $49,4 \mathrm{~B}$ & Não significativa & - \\
\hline & 8 & $21,4 \mathrm{~A}$ & $18,5 \mathrm{~A}$ & $20,2 \mathrm{~A}$ & $20,0 \mathrm{~A}$ & Não significativa & - \\
\hline & 9 & $43,3 \mathrm{~B}$ & $40,9 \mathrm{~B}$ & $48,9 \mathrm{BC}$ & $51,5 \mathrm{BC}$ & $y=38+0,5433 x^{* *}$ & 0,74 \\
\hline & 10 & $57,7 \mathrm{C}$ & $51,3 \mathrm{C}$ & $57,8 \mathrm{C}$ & $59,8 \mathrm{C}$ & \multirow[t]{2}{*}{ Não significativa } & \multirow[t]{2}{*}{-} \\
\hline & & & $\mathrm{CV}(\%)=$ & & & & \\
\hline
\end{tabular}

Médias seguidas da mesma letra maiúscula, na coluna, não diferem entre si, pelo teste Tukey ( $\mathrm{p} \leq 0,05)$. ** Significativo a 5\% (Teste F).

Nesse sentido, considerando-se que a diferença entre a temperatura de embebição e de avaliação pode influenciar nos resultados, recomenda-se o uso de $25^{\circ} \mathrm{C}$, para a condução do teste de condutividade elétrica, pois esta temperatura permitiu a estratificação de ambas as cultivares, quanto à qualidade fisiológica, e, também, por ser esta a temperatura mais próxima das condições ambientais de laboratório. Assim, os resultados obtidos levam a sugerir que se deve ter cautela na interpretação dos valores de condutividade elétrica da solução de embebição de sementes de girassol. A utilização de quatro subamostras de 50 sementes puras, embebidas em $75 \mathrm{~mL}$ de água e mantidas a $25^{\circ} \mathrm{C}$, foi a melhor opção, pois permitiu estratificar os lotes, quanto ao vigor, em todos os períodos avaliados.

\section{CONCLUSÃO}

As cultivares de sementes de girassol Catissol e Multissol apresentaram resultados semelhantes para o teste de condutividade elétrica, sendo a combinação de 50 sementes, em $75 \mathrm{~mL}$ de água, a $25^{\circ} \mathrm{C}$, nos períodos de 18 e 24 horas de embebição, a mais promissora para a avaliação da sua qualidade fisiológica.

\section{REFERÊNCIAS}

ABREU, L. A. S. et al. Teste de condutividade elétrica na avaliação de sementes de girassol armazenadas sob diferentes temperaturas. Revista Brasileira de Sementes, Brasília, DF, v. 33, n. 4, p. 637-644, 2011.

ALBUQUERQUE, M. C. F. et al. Testes de condutividade elétrica e de lixiviação de potássio na avaliação da qualidade fisiológica de sementes de girassol. Revista Brasileira de Sementes, Brasília, DF, v. 23, n. 1, p. 1-8, 2001.

ALVES, E. et al. Efeito dos períodos de envelhecimento na lixiviação de íons e proteínas solúveis em sementes de milho. Revista Brasileira de Sementes, Brasília, DF, v. 26, n. 2, p. 119-125, 2004. 
ARAÚJO, R. F. et al. Teste de condutividade elétrica para sementes de pinhão-manso (Jatropha curcas L.). Idesia, Arica, v. 29, n. 2, p. 79-86, 2011.

\section{ASSOCIATION OF OFFICIAL SEED ANALYSTS} (AOSA). Seed vigor testing handbook. East Lasing: AOSA, 1983. (Contribution, 32).

BARBIERI, A. P. P. et al. Teste de lixiviação de potássio para a avaliação do vigor de sementes de arroz. Revista Brasileira de Sementes, Viçosa, MG, v. 34, n. 1, p. 117 124, 2012.

BRANDÃO JÚNIOR, D. S. et al. Adequação do teste de condutividade elétrica para determinar a qualidade fisiológica de sementes de girassol. Informativo Abrates, Londrina, v. 17, n. 1/2, p. 184, 1997.

BRASIL. Ministério da Agricultura, Pecuária e Abastecimento. Secretaria de Defesa Agropecuária. Regras para análise de sementes. Brasília, DF: MAPA/ ACS, 2009.

COlete, J. C. F.; VIEIRA, R. D.; DUTRA, A. S. Electrical conductivity and soybean seedling emergence. Scientia Agricola, Piracicaba, v. 61, n. 4, p. 386-391, 2004.

COSTA, P. S. C.; CARVAlHO, M. L. M. Teste de condutividade elétrica individual na avaliação da qualidade fisiológica de sementes de café. Ciência e Agrotecnologia, Lavras, v. 30, n. 1, p. 92-96, 2006.

DIAS, D. C. F. S.; MARCOS FILHO, J. Testes de condutividade elétrica para avaliação do vigor de sementes de soja (Glycine Max (L.) Merrill). Scientia Agricola, Piracicaba, v. 53, n. 1, p. 31-42, 1996.

DIAS, D. C. F. S.; MARCOS FILHO, J.; CARMELLO, Q. A. C. Potassium leakage test for the evaluation of vigour in soybean seeds. Seed Science and Technology, Zürich, v. 25, n. 1, p. 7-18, 1996.

FAGUNDES, M. H. Sementes de girassol: alguns comentários. Brasília, DF: MAPA/Conab/SUGOF, 2002.

FERREIRA, D. F. Sisvar: um programa para análises e ensino de estatística. Symposium, Lavras, v. 6, n. 2, p. 3641, 2008.

HALDER, S.; GUPTA, K. Effect of storage of sunflower seeds in high and low relative humidity on solute leaching and internal biochemical changes. Seed Science and Technology, Zürich, v. 8, n. 1, p. 317-321, 1980.

HAMPTON, J. G.; TEKRONY, B. M. Conductivity test. In: HAMPTON, J. G.; TEKRONY, B. M. (Eds.). Handbook of vigour methods. 3. ed. Zürich: ISTA, 1995. p. 22-34.

KRZYZANOWSKI, F. C.; FRANÇA NETO, J. B.; HENNING, A. A. Relato dos testes de vigor disponíveis para as grandes culturas. Informativo Abrates, Brasília, DF, v. 1, n. 2, p. 15-50, 1991.
KRZYZANOWSKI, F. C.; VIEIRA, R. D.; FRANÇA NETO, J. B. Vigor de sementes: conceitos e testes. Londrina: Abrates, 1999.

LOEFFLER, T. M.; TEKRONY, D. M.; EGLI, B. D. The bulk conductivity test as an indicator of soybean seed quality. Journal of Seed Technology, Springfield, v. 12, n. 1, p. 37-53, 1988.

MARCOS FILHO, J. Teste de envelhecimento acelerado. In: KRZYZANOWSKI, F. C.; VIEIRA, R. D.; FRANÇA NETO, J. B. (Eds.). Vigor de sementes: conceitos e testes. Londrina: Abrates, 1999. p. 1-24.

MARCOS FILHO, J. et al. Estudo comparativo de métodos para avaliação da qualidade fisiológica de sementes de soja, com ênfase ao teste de condutividade elétrica. Pesquisa Agropecuária Brasileira, Brasília, DF, v. 25, n. 12, p. 1805-1815, 1990.

NAKAGAWA, J. Testes de vigor baseados na avaliação das plântulas. In: VIEIRA, R. D.; CARVALHO, N. M. Testes de vigor em sementes. Jaboticabal: Funep/Unesp, 1994. p. 49-86.

NIJÊNSTEIN, H. et al. ISTA handbook on moisture determination. Basserdorf: International Seed Testing Association, 2007.

OLIVEIRA, A. R. S.; NOVEMBRE, A. D. L. C. Teste de condutividade elétrica para as sementes de pimentão. Revista Brasileira de Sementes, Brasília, DF, v. 27, n. 1, p. 31-36, 2005.

QUEIROGA, V. P.; DURAN, J. M. Avaliação da condutividade elétrica em sementes de girassol (Helianthus annuus L.) com diferentes graus de umidade. Informativo Abrates, Brasília, DF, v. 7, n. 1/2, p. 176, 1997.

SCHEEREN, B. R. et al. Qualidade fisiológica e produtividade de sementes de soja. Revista Brasileira de Sementes, Brasília, DF, v. 32, n. 3, p. 35-41, 2010.

SILVA, L. B.; MARTINS, C. C. Teste de condutividade elétrica para sementes de mamoneira. Ciências Agrárias, Londrina, v. 30, supl., p. 1043-1050, 2009.

SOUZA, L. A. et al. Teste de condutividade elétrica para avaliação da qualidade fisiológica de sementes de mamona. Revista Brasileira de Sementes, Brasília, DF, v. 31, n. 1, p. 60-067, 2009.

TORRES, S. B. Comparação entre testes de vigor para avaliar a qualidade fisiológica de sementes de algodão. Revista Brasileira de Sementes, Brasília, DF, v. 20, n. 2, p. 11-15, 1998.

UNGARO, M. R. G. Potencial da cultura do girassol como fonte de matéria-prima para o Programa Nacional de Produção e Uso do Biodiesel. In: UNGARO, M. R. G. Agronegócio de plantas oleaginosas: matérias-primas para biodiesel. Piracicaba: ESALQ, 2006. p. 57-80. 
VANZOLINI, S.; NAKAGAWA, J. Teste de condutividade elétrica em sementes de amendoim. Revista Brasileira de Sementes, Brasília, DF, v. 27, n. 2, p. 151-158, 2005.

VANZOLINI, S.; NAKAGAWA, J. Teste de condutividade elétrica em sementes de amendoim: efeitos de teor de água inicial e de período de embebição. Revista Brasileira de Sementes, Brasília, DF, v. 21, n. 1, p. 46-52, 1999.
VIEIRA, R. D.; KRZYZANOWSKI, F. C. Teste de condutividade elétrica. In: KRZYZANOWSKI, F. C.; VIEIRA, R. D.; FRANÇA NETO, J. B. (Eds). Vigor de sementes: conceitos e testes. Londrina: Abrates, 1999. p. 1-26.

VIEIRA, R. D. et al. Electrical conductivity of the seed soaking solution and soybean seedling emergence. Scientia Agricola, Piracicaba, v. 61, n. 2, p. 164-168, 2004. 